\title{
O Desafio da Utilização de Tecnologias Computacionais para Suporte a Crianças com Transtorno do Espectro Autista
}

\author{
Patrícia S. Almeida Ivo', Ivana Cristina H. C. Barreto², David Viana ${ }^{3}$, Luiz \\ Odorico M. Andrade ${ }^{2}$, Mauro Oliveira ${ }^{3}$ \\ ${ }^{1}$ Secretaria Municipal de Saúde de Fortaleza - Rua do Rosário, 283 - CEP: 60055-090 \\ Fortaleza, CE - Brasil \\ ${ }^{2}$ Fundação Oswaldo Cruz - Eusébio, CE - Brasil \\ ${ }^{3}$ Instituto Federal de Educação, Ciência e Tecnologia do Ceará (IFCE) - Aracati, CE - \\ Brasil

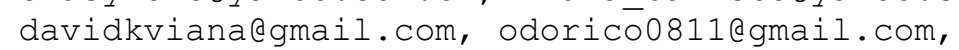

\begin{abstract}
Autistic Spectrum Disorder (ASD) is among the most damaging mental health problems in child development. In general, people with autism need language, behavioral, and social skills support to be successful. The present study sought to know the main challenges faced by children with ASD and their families / caregivers, based on the difficulties reported by the families of these children and by the multi-professional team that accompanies them. The results found may support future research aimed at mitigating these needs.

Resumo. O Transtorno do Espectro Autista (TEA) está entre os problemas de saúde mental que mais prejudicam o desenvolvimento infantil. Em geral, pessoas com o autismo precisam de suporte de habilidades linguísticas, comportamentais e sociais para serem bem-sucedidas. O presente trabalho buscou conhecer os principais desafios vivenciados por crianças com TEA $e$ seus familiares/cuidadores, com base nas dificuldades relatadas pelos próprios familiares destas crianças e pela equipe multiprofissional que as acompanha. Os resultados encontrados podem subsidiar pesquisas futuras voltadas à atenuação dessas necessidades.
\end{abstract}

\section{Introdução}

O Transtorno do Espectro Autista (TEA) está entre os problemas de saúde mental que mais prejudicam o desenvolvimento infantil [A.P.A., 2013]. Dados do Centro de Desenvolvimento Infantil (CDC) de Harvard mostram a cifra de 1:59 como sendo a prevalência de TEA entre crianças de 8 anos de idade, em 11 cidades dos EUA [BAIO, 2018]. No Brasil, até o momento, foi realizado um único estudo (piloto) estimando a prevalência de TEA em $0,3 \%$ [Paula et al., 2011]. A partir deste estudo, associado a dados internacionais, é possível estimar que, aproximadamente, 1,5 a 2 milhões de brasileiros tenham TEA, o que aponta para a relevância do tema.

Segundo Ennis-Cole e Smith (2011), a população de indivíduos com TEA é heterogênea, cada indivíduo tendo suas próprias singularidades, porém existem 
denominadores comuns: déficits em habilidades de comunicação e sociais, além de comportamentos restritivos e / ou repetitivos. De acordo com Brentani et al. (2013), aproximadamente $60-70 \%$ das pessoas com TEA têm algum nível de deficiência intelectual. Em geral, pessoas com o autismo precisam de suporte de habilidades linguísticas, comportamentais e sociais para serem bem-sucedidas [Ennis-Cole e Smith, 2011].

Neste sentido, os autores deste trabalho, realizaram um estudo qualitativo [Minayo, 2013], descritivo, exploratório, e transversal, com familiares, cuidadores, e profissionais que atendem crianças com TEA. Foram realizados três grupos focais (total de 25 participantes) com familiares/cuidadores de crianças com TEA de seis a doze anos de idade incompletos, na Casa da Esperança, uma fundação sem fins lucrativos, referência no atendimento de pessoas com TEA, situada em Fortaleza (Brasil). Nos grupos focais foram relatados os desafios enfrentados pelas crianças e familiares no cotidiano e suas potencialidades, a maneira pela qual suas crianças já utilizam a tecnologia na rotina diária e sugestões de possíveis aplicações de Tecnologias de Informação e Comunicação (TIC'S). Também foram entrevistados seis profissionais de nível superior da saúde e da educação da instituição sobre estes tópicos.

Constatou-se, no universo dos participantes do estudo, que as crianças autistas têm, de uma maneira geral, facilidade para o aprendizado através de figuras e para o uso de ferramentas tecnológicas. A maioria das crianças do estudo apresentou uma maior dependência em relação às atividades de vida diária (AVD'S) e maior dificuldade em aprendê-las, sendo este um dos principais desafios relatados por familiares e profissionais de apoio ao TEA. Além disso, muitos familiares/cuidadores, em especial as mães, relataram que tiveram que abandonar seus empregos para cuidar de suas crianças e externaram o sentimento de solidão e falta de apoio, em muitas ocasiões, necessitando serem apoiadas, assim como seus filhos. Também relataram a necessidade de fonte segura de informações onde possam aprender mais sobre o TEA, favorecendo o desenvolvimento de suas crianças.

\section{Descrição do problema}

As AVD'S são habilidades aprendidas na infância e incluem atividades de autocuidado e higiene pessoal, como ir ao banheiro, tomar banho, escovar os dentes, alimentar-se, vestirse e comer. Elas são fundamentais para a adaptação, a sobrevivência e o convívio adequado do indivíduo em ambiente social [GUIMARAES e CARMO, 2018]. O aprendizado, que para as crianças com desenvolvimento neuropsicomotor normal ocorre quase que naturalmente, através da imitação de adultos ou de seus pares nas atividades da vida diária, para a criança com TEA, a depender do grau do autismo, representa algo a ser conquistado com treino exaustivo e que, algumas vezes, não se alcança. $\mathrm{O}$ relato a seguir de uma participante do estudo expressa essa dificuldade: "É uma luta diária. É como se todos os dias eu tivesse que ensinar a mesma coisa pra ele nunca esquecer do que está fazendo...”.

A dependência das crianças com TEA para o aprendizado das atividades da vida diária ocorre, dentre outras causas, pela ausência ou diminuição da capacidade de imitar, que é um dos pré-requisitos cruciais para o aprendizado. Além disso, eles apresentam, frequentemente, dificuldades na coordenação motora tanto grossa como fina, dificuldades 
na organização e planejamento da execução de tarefas e na generalização do aprendizado. [BRUNONI; MERCADANTE; SCHWARTZMAN, 2014].

A tecnologia não substitui as intervenções educativas que familiares e terapeutas executam, mas poderia dar um suporte na questão da esquematização das etapas das AVD'S, assim como na possibilidade de repetição quantas vezes fossem necessárias do modo de realização dessas atividades. Dessa forma, também poderia auxiliar a diminuir a sobrecarga dos familiares.

Deve-se ter em mente que o autista faz parte de uma determinada família, que possui características singulares e, para que se possa de fato beneficiá-la, é preciso que os caminhos propostos para ele também acolham a sua família. A família de uma criança autista necessita tanto de atendimento e orientação quanto o próprio indivíduo, não só para sua própria organização e ajustamento, como também para que possa constituir um elemento de apoio e ajuda ao processo de educação e reabilitação [PINTO et al., 2016].

\section{Relevância}

O contexto familiar sofre rupturas imediatas diante do início da apresentação dos sintomas de TEA na medida em que há interrupção das atividades rotineiras e transformação do clima emocional no qual a família vive. Na revisão de literatura de Andrade e Teodoro (2012) encontrou-se que a união e a mobilização da família em torno das dificuldades de sua criança são determinantes no início da adaptação. No caso de crianças com algum transtorno do desenvolvimento, o impacto das dificuldades inerentes sobre a família vai depender de uma complexa interação entre a gravidade dos sintomas da criança e as características psicológicas dos pais, tais como resiliência, estilo de enfrentamento, bem como a disponibilidade de recursos comunitários e sociais.

O autismo também causa um impacto sobre as famílias do ponto de vista econômico. Pouquíssimas famílias têm condições econômicas de arcar com o custo do tratamento adequado e, para atender as necessidades geradas pelo autismo todas elas dependerão, em algum momento, de algum tipo de apoio institucional.

Embora tenham ocorrido avanços nos direitos da pessoa com TEA na legislação brasileira, as ofertas de cuidados profissionais abrangentes sem desembolso direto das famílias são raras. É necessário a ampliação de políticas sociais, bem como o desenvolvimento de ferramentas tecnológicas para o suporte à pessoa com TEA e sua família, sem o que estas podem ficar à margem da sociedade, em um ciclo de isolamento e empobrecimento.

O desenvolvimento de tecnologias qualificadas para suporte às crianças com TEA e seus familiares/cuidadores pode ser bastante relevante para a promoção de saúde deste grupo de pessoas. A Lei Brasileira de Inclusão (LBI), nº 13.146, de 6 de julho de 2015, em seu artigo 78 orienta que: "Devem ser estimulados a pesquisa, o desenvolvimento, a inovação e a difusão de tecnologias voltadas para ampliar o acesso da pessoa com deficiência às tecnologias da informação e comunicação e às tecnologias sociais" [Brasil, 2015, p.7].

\section{Mercado/Público Alvo}

A princípio, o público alvo que pode se beneficiar das soluções dos desafios encontrados são as pessoas com TEA, seus familiares e/ou cuidadores, além de organizações 
filantrópicas, públicas e privadas que oferecem atenção multiprofissional às pessoas com TEA, com suas equipes, em geral, envolvendo neurologista, psiquiatra, psicólogo, terapeuta ocupacional, psicopedagogo, fonoaudiólogo, fisioterapeuta, dentre outros.

A escola, incluindo professores, diretores e toda a equipe de funcionários, também é uma instituição interessada no desenvolvimento da criança com TEA, portanto, possível público alvo.

Para o Sistema Único de Saúde (SUS) e para os convênios de saúde privados também é interessante a solução ou atenuação dos desafios relatados.

\section{Soluções existentes}

O pensamento por imagens é uma característica marcante nos indivíduos com TEA e funciona como uma forma de absorção de conhecimento [GRANDIN, 2012]. Além disso, indivíduos no espectro autista apresentam muitas dificuldades ao realizarem atividades complexas e abstratas. Essas dificuldades podem ser minimizadas com o uso de instruções visuais, sendo importante ensinar uma coisa de cada vez, de forma sequencial, para que haja a compreensão das crianças daquilo que deve ser realizado [GRANDIN, 2012].

Atualmente existem ferramentas físicas já utilizadas no aprendizado da criança com TEA, como por exemplo, cartazes indicando desenhos de passo a passo de uma AVD (lavar as mãos, escovar os dentes, tomar banho, usar o vaso sanitário para necessidades fisiológicas, dentre outras). No entanto, essas ferramentas são muitas vezes baseadas em papel, o que as torna volumosas, podendo ser inaceitáveis ou impraticáveis em muitos contextos. A compacidade seria outra característica favorecedora do emprego de TIC'S por pessoas com TEA.

Em relação a tecnologias para o treinamento das atividades de vida diária, Bimbrahw, Boger e Mihailidis (2012) desenvolveram um dispositivo computadorizado que foi usado por crianças com TEA e seus cuidadores, para ajudar de forma autônoma com atividades de autocuidado (lavar as mãos), em um estudo piloto. O dispositivo usou visão computacional e inteligência artificial para rastrear uma criança com TEA através de uma atividade, e emitia avisos de áudio e visual conforme necessário. Os resultados mostraram que o dispositivo respondeu corretamente a aproximadamente $74 \%$ das situações que encontrou. Apesar dos bons resultados, o dispositivo idealizado apresenta uma solução de ferramenta tecnológica de alto custo para o consumidor e não escalável.

O jogo ACA (Aprendendo com Comunicação Alternativa), desenvolvido por Gobbo et al. (2018), apresenta um aplicativo gamificado para a alfabetização de crianças com TEA. Ele conta com o Picture Exchange Communication System (PECS), um sistema de comunicação alternativa por troca de figuras, empregando pictografias relacionadas às AVD'S, para que estas possam se familiarizar com as atividades que têm que realizar todos os dias. Entretanto não é exposto o passo a passo das AVD'S, apenas uma figura única que representa aquela atividade.

O aplicativo "Autismo Projeto Integrar" se propõe a ser uma ferramenta de apoio no desenvolvimento e aprendizado das atividades da vida diária de pessoas com TEA. Esse aplicativo foi projetado para celulares e tablets que utilizam sistema Android e pode ser baixado gratuitamente na plataforma Google Play Store (KRAUSE, 2016). Ele foi elaborado com base no método dos "Desenhos Roteirizados" do site Autismo Projeto 
Integrar. Como limitações desse aplicativo, temos que algumas atividades de vida diária ainda não estão contempladas. Além disso, os familiares/cuidadores provavelmente sempre precisarão apoiar o uso do mesmo, pois não apresenta atrativos para que as crianças com TEA procurem seu uso espontaneamente.

Segundo os participantes dos grupos focais realizados pelos autores do presente estudo, quando os aplicativos não oferecem conteúdo que seja estimulante para as crianças, com temas de suas preferências, o tempo de uso é bem curto. O desafio é a construção de uma aplicação que possa ser atrativa, considerando as preferências e habilidades existentes das crianças e familiares para o sucesso das intervenções tecnológicas.

Em relação a aplicações para apoiar familiares e cuidadores de pessoas com TEA, não se encontrou na revisão bibliográfica soluções existentes sobre este aspecto. Uma aplicação que pudesse apoiar o aprendizado dos familiares sobre o autismo, visto que a falta de conhecimento sobre o assunto, principalmente na suspeita e no momento inicial do diagnóstico, e de uma fonte confiável para se buscar informações corretas, foram queixas frequentes nos grupos focais. A família é uma peça fundamental no desenvolvimento do indivíduo com TEA e ela precisa estar capacitada e ao mesmo tempo ter apoio para que isso possa ocorrer da melhor forma.

\section{Conclusão}

O presente estudo traz conhecimentos sobre quais desafios ao desenvolvimento das crianças com TEA são importantes na construção de instrumento de TIC'S, segundo o olhar de familiares e/ou cuidadores destas crianças e da equipe multiprofissional, sendo a dependência nas atividades da vida diária o principal desafio relatado.

A necessidade de uma aplicação de suporte aos familiares de indivíduos com TEA foi um resultado que surpreendeu aos autores da pesquisa, que inicialmente, esperavam que seriam relatados desafios do cotidiano especialmente das crianças com TEA.

$\mathrm{O}$ conhecimento desses desafios pode subsidiar pesquisas futuras voltadas à atenuação destas dificuldades. A pesquisa tecnológica para indivíduos com TEA deve combinar diversos saberes: dos tecnólogos, de designers, da equipe multiprofissional, educadores, pesquisadores biomédicos, dos familiares e dos próprios indivíduos com TEA, participando na construção de um melhor futuro para estes indivíduos e seus familiares e/ou cuidadores.

A Casa da Esperança, uma fundação sem fins lucrativos, que atende cerca de 400 pessoas com autismo em regime intensivo, de quatro ou oito horas por dia, com sede em Fortaleza (Ceará), é uma organização onde poderão ser realizados testes para uma ferramenta tecnológica a ser desenvolvida, apresentando infraestrutura adequada e profissionais de saúde qualificados, que apresentam abertura ao uso de novas tecnologias.

\section{Referências}

American Psychiatric Association - A.P.A. (2013), Diagnostic and Statistical Manual of Mental Disorders (DSM-V) 5a . ed. Washington.

Andrade, A. A. e Teodoro, M. L. (2012). Família e Autismo: Uma Revisão da Literatura. Contextos Clínicos, vol. 5, n. 2, p. 133-142. 
Baio, J. et al. (2018). Prevalence of Autism Spectrum Disorder Among Children Aged 8 Years - Autism and Developmental Disabilities Monitoring Network, 11 Sites, United States, 2014. MMWR Surveill Summ 2018, v. 67, n. 6, p.1-23.

Bimbrahw, J., Boger, J. and Mihailidis, A. (2012). Investigating the Efficacy of a Computerized Prompting Device to Assist Children with Autism Spectrum Disorder with Activities of Daily Living. Assistive Technology, v.24, n.4, p. 286-298.

Brasil (2015). Lei n. 13.146, de 6 de julho de 2015. Institui a Lei Brasileira de Inclusão da Pessoa com Deficiência (Estatuto da Pessoa com Deficiência). Diário Oficial da União. 6 jul 2015.

Brentani, H. et al. (2013). Autism spectrum disorders: an overview on diagnosis and treatment. Rev. Bras. Psiquiatr., São Paulo, v. 35, supl. 1, p. S62-S72.

Brunoni, D.; Mercadante, M. T.; Schwartzman, J. S. (2014). Transtornos do Espectro do Autismo. In: LOPES, A. C. (Org.) Clínica Médica: diagnóstico e tratamento. 1.ed. São Paulo: Atheneu, p. 5731-5746.

Ennis-Cole, D. and Smith, D. (2011). Assistive technology and autism: Expanding the technology leadership role of the school librarian. School Libraries Worldwide, v.17, n. 2.

Gobbo, M. R. M. et al. (2018). Jogo ACA para indivíduos com Transtorno do Espectro Autista. In: SBGames, 17., 2018, Foz do Iguaçu - PR - Brasil. Proceedings of SBGames 2018. Foz do Iguaçu: SBGames, p. 1114-1121.

Grandin, T. (2002). Teacching tips for children and adults with autism. Indiana University Bloomington, dec. 2002. Disponível em: <http://www.iidc.indiana.edu/index.php?pageId=601>. Acesso em: 20 mar. 2019.

Guimaraes, L. M.; Carmo, J. S. (2018). Procedimentos eficazes no treino de toalete para indivíduos com autismo: contribuições de Azrin e colaboradores. Rev. Psicol. Saúde, Campo Grande, v. 10, n. 2, p. 69-82, ago. 2018.

Krause, M. (2016). Apresentação. Site do autor, 27 ago. 2016. Disponível em: <http://www.maicokrause.com/>. Acesso em: 23 Mar. 2019.

Minayo, M.C. (2013) O desafio do conhecimento: pesquisa qualitativa em saúde. 14a . ed. São Paulo: Hucitec.

Paula C. S. et al. (2011). Brief report: prevalence of pervasive developmental disorder in Brazil: a pilot study. J Autism Dev Disord, v. 41, n.12, p. 1738-42.

Pinto, R. N. M. et al. (2016). Autismo infantil: impacto do diagnóstico e repercussões nas relações familiares. Rev. Gaúcha Enferm. Porto Alegre, v. 37, n. 3, e61572. 